\title{
Extended Application Of Time-Varying Observer For Rigid Rotors Unbalance Estimation During Acceleration
}

\author{
Xunxing $\mathrm{Yu}^{1, \mathrm{a}}$, Kuanmin $\mathrm{Mao}^{1}$ and Yaming $\mathrm{Zhu}^{1}$ \\ ${ }^{1}$ School of Mechanical Science and Engineering, Huazhong University of Science and Technology, Wuhan, Hubei, \\ 430074, China
}

\begin{abstract}
Unbalance is one of essential problems for modern rotating machines. In this work, an improved time-varying observer is proposed to estimate the unbalance of rigid rotor during acceleration. In order to fitting different speed acceleration laws, the unbalance forces have been included in an new designed augmented states, meanwhile the state space model of rigid rotor has been also developed. The developed state space model is transformed to a canonical transformation and a new designed time-varying observer can be obtained. The estimated unbalances can be directly obtained by using this time-varying observer. This method would be very helpful for active balancing control strategy during acceleration.
\end{abstract}

\section{Introduction}

Modern rotating machines are designed highly-intelligent and highly-integration with speeds-up to achieve excellent quality and efficiency. Excessive vibration would deduced by unbalance forces which are proportional to the square of the spin speed, thus unbalance is an essential problem in rotordynamics.

Traditional off-line balancing was always taken after the system power off, which is timeconsuming and costly. In order to perform balancing scheme without interrupting the operation of the rotor system, researchers have done valuable works for speed-varying situation. Active magnetic bearings have been applied in active balancing system for the attenuation of unbalance induced responses during speed-varying stage by interpolate the influence coefficients between different speeds [1]. Mass redistribution balancers using gain scheduling strategy during acceleration have been conducted based on instantaneous influence coefficients, which are calculated from the fileted speedsynchronous response [2]. However, the non-synchronous vibration excited by the acceleration might cause difficulties if the rotor system is in high acceleration or small damping when applying the above method.

An alternative way to estimate the system unbalance by using time-varying observer for rigid rotor system during acceleration. The estimated result can be used in active balancing for rigid rotors [3]. However, this method is based on constant acceleration assumption. In order to applying this method for different speed laws such as exponential law, a new method is proposed by developed the dynamic model.

The main purpose of this paper is to improving the adaption the time-varying observer for different spin acceleration laws. An improved rigid model for different spin acceleration laws will be presented

${ }^{a}$ Corresponding author: yuxx@hust.edu.cn 
in section 2, the procedures of the time-varying observer design is given in section 3. A simulation is used to validate the performance of the proposed method. The results shown that the proposed method could fitting different speed acceleration laws.

\section{Dynamic Model of Rigid Rotors}

For many practical rotors such as spindles are usually operating below the first critical speed for accuracy and safety, moreover, flexible rotors also need to balancing in low speeds, in such conditions, four freedom rigid rotor model can be used. The rigid rotors model is shown in figure 1.
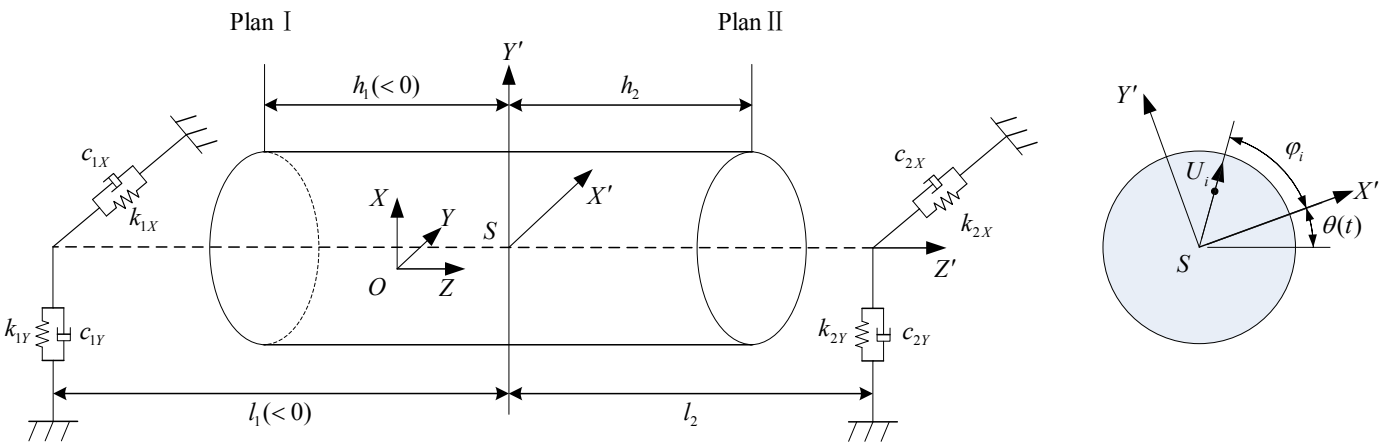

Figure 1. Four freedom rigid rotor model.

As shown in figure $1, S$ is the mass center of the rigid rotor, $S X^{\prime} Y^{\prime} Z^{\prime}$ is the rotor-fixed frame and $O X Y Z$ is the inertial frame, the $S X^{\prime} Y^{\prime} Z^{\prime}$ coordinate system is coincides with the $O X Y Z$ coordinate system when the rotor is at rest. The bearings are modelled as a set of linear springs and dampers in two orthogonal directions. Two unbalances in plan I and plan II are used to represent the inherent dynamic unbalance of the rotor. The motions are described by displacements $x_{G}, y_{G}$ of the mass center with respect to the inertial reference, as well as the small angles $\alpha$ and $\beta$ rotation about the $X$ and $Y$ axis.

The dynamic equation of motion of a rigid body can be obtained [4]

$$
\left\{\begin{array}{l}
m \ddot{x}_{G}+k_{1 X} x_{1}+c_{1 X} \dot{x}_{1}+k_{2 X} x_{2}+c_{2 X} \dot{x}_{2}=\operatorname{Re}\left(-\frac{d^{2}}{d t^{2}} e^{j \theta} \sum_{i=1}^{2} U_{i}\right) \\
m \ddot{y}_{G}+k_{1 Y} y_{1}+c_{1 Y} \dot{y}_{1}+k_{2 Y} y_{2}+c_{2 Y} \dot{y}_{2}=\operatorname{Im}\left(-\frac{d^{2}}{d t^{2}} e^{j \theta} \sum_{i=1}^{2} U_{i}\right) \\
J_{t} \ddot{\alpha}+J_{p} \dot{\theta} \dot{\beta}+J_{p} \ddot{\theta} \beta-\left(k_{1 Y} y_{1}+c_{1 Y} \dot{y}_{1}\right) l_{1}-\left(k_{2 Y} y_{2}+c_{2 Y} \dot{y}_{2}\right) l_{2}=\operatorname{Im}\left(\frac{d^{2}}{d t^{2}} e^{j \theta} \sum_{i=1}^{2} U_{i} h_{i}\right) \\
J_{t} \ddot{\beta}-J_{p} \dot{\theta} \dot{\alpha}-J_{p} \ddot{\theta} \alpha+\left(k_{1 X} x_{1}+c_{1 X} \dot{x}_{1}\right) l_{1}+\left(k_{2 X} x_{2}+c_{2 X} \dot{x}_{2}\right) l_{2}=\operatorname{Re}\left(-\frac{d^{2}}{d t^{2}} e^{j \theta} \sum_{i=1}^{2} U_{i} h_{i}\right)
\end{array}\right.
$$

where $\operatorname{Re}(\bullet)$ and $\operatorname{Im}(\bullet)$ means the real and imaginary part of a complex number, $j=\sqrt{-1}$, $U_{i}=\left|U_{i}\right| e^{j \varphi_{i}}(i=1,2)$ are complex numbers as unbalances in the coordinate system, $J_{p}$ and $J_{t}$ and the diametric moments of inertia of the rotor, respectively. $\theta(t)$ is the time-varying spin speed. $x_{1}$, $y_{1}, x_{2}, y_{2}$ represent the displacements at the location of the bearings in the inertial frame.

Here exist such relation that 
Substitute equation (2) into (1)

$$
\left[\begin{array}{l}
x_{1} \\
x_{2} \\
y_{1} \\
y_{2}
\end{array}\right]=\left[\begin{array}{cccc}
1 & 0 & 0 & l_{1} \\
1 & 0 & 0 & l_{2} \\
0 & 1 & -l_{1} & 0 \\
0 & 1 & -l_{2} & 0
\end{array}\right]\left[\begin{array}{c}
x_{G} \\
y_{G} \\
\alpha \\
\beta
\end{array}\right]
$$

$$
\mathbf{M} \ddot{\mathbf{q}}+(\mathbf{C}+\dot{\theta} \mathbf{G}) \dot{\mathbf{q}}+(\mathbf{K}+\ddot{\theta} \mathbf{G}) \mathbf{q}=\mathbf{F}
$$

where $\mathbf{q}=\left[\begin{array}{llll}x_{G} & y_{G} & \alpha & \beta\end{array}\right]^{T}$, matrices $\mathbf{M}, \mathbf{C}, \mathbf{G}, \mathbf{K}, \mathbf{F}$ are the mass, damping, gyroscopic, stiffness matrices and unbalance forces vector, and are given as

$$
\begin{array}{r}
\mathbf{M}=\left[\begin{array}{cccc}
m & 0 & 0 & 0 \\
0 & m & 0 & 0 \\
0 & 0 & J_{t} & 0 \\
0 & 0 & 0 & J_{t}
\end{array}\right], \mathbf{G}=\left[\begin{array}{cccc}
0 & 0 & 0 & 0 \\
0 & 0 & 0 & 0 \\
0 & 0 & 0 & J_{p} \\
0 & 0 & -J_{p} & 0
\end{array}\right], \mathbf{F}=\left[\begin{array}{c}
\operatorname{Re}\left(f_{1}\right) \\
\operatorname{Im}\left(f_{1}\right) \\
\operatorname{Re}\left(f_{2}\right) \\
\operatorname{Im}\left(f_{2}\right)
\end{array}\right] \\
\mathbf{C}=\left[\begin{array}{cccc}
c_{1 x}+c_{2 x} & 0 & 0 & c_{1 x} l_{1}+c_{2 x} l_{2} \\
0 & c_{1 y}+c_{2 y} & -c_{1 y} l_{1}-c_{2 y} l_{2} & 0 \\
0 & -c_{1 y} l_{1}-c_{2 y} l_{2} & c_{1 y} l_{1}^{2}+c_{2 y} l_{2}^{2} & 0 \\
c_{1 x} l_{1}+c_{2 x} l_{2} & 0 & 0 & c_{1 x} l_{1}^{2}+c_{2 x} l_{2}^{2}
\end{array}\right] \\
\mathbf{K}=\left[\begin{array}{cccc}
k_{1 x}+k_{2 x} & 0 & 0 & k_{1 x} l_{1}+k_{2 x} l_{2} \\
0 & k_{1 y}+k_{2 y} & -k_{1 y} l_{1}-k_{2 y} l_{2} & 0 \\
0 & -k_{1 y} l_{1}-c_{2 y} l_{2} & k_{1 y} l_{1}^{2}+k_{2 y} l_{2}^{2} & 0 \\
k_{1 x} l_{1}+k_{2 x} l_{2} & 0 & 0 & k_{1 x} l_{1}^{2}+k_{2 x} l_{2}^{2}
\end{array}\right]
\end{array}
$$

where $f_{1}=C_{1} \frac{d^{2}}{d t^{2}} e^{j \theta}, f_{2}=C_{2} \frac{d^{2}}{d t^{2}} e^{j \theta}, C_{1}$ and $C_{2}$ are complex numbers and defined as $C_{1}=-\sum_{i=1}^{2} U_{i}, C_{2}=j \sum_{i=1}^{2} U_{i} h_{i}$.

The angular acceleration law of the rotational motion may be not constant, such as exponential law, in order to corporates with different kinds of speed-varying law, an complex number is introduced as $g=e^{j \theta}$ and

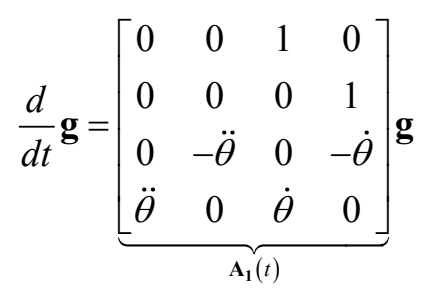

where $\mathbf{g}=\left[\begin{array}{llll}\operatorname{Re}(g) & \operatorname{Im}(g) & \operatorname{Re}(\dot{g}) & \operatorname{Im}(\dot{g})\end{array}\right]^{T}$, obviously, $f_{1}$ and $f_{2}$ are the results of $\ddot{g}$ multiplied by complex constants $C_{1}$ and $C_{2}$ relatively, complex variables $g_{1}=C_{1} g$ and $g_{2}=C_{2} g$ also satisfy equation (7). For the sake of convenience, two vectors are defined as

Then representing the rigid rotor system into the state space model by including the unbalance force $\mathbf{F}$ to the state vector 


$$
\begin{aligned}
& \frac{d \mathbf{z}}{d t}=\underbrace{\left[\begin{array}{cccc}
\mathbf{0} & \mathbf{I}_{4 \times 4} & \mathbf{0} & \mathbf{0} \\
-\mathbf{M}^{-1}(\mathbf{K}+\ddot{\theta} \mathbf{G}) & -\mathbf{M}^{-1}(\mathbf{C}+\dot{\theta} \mathbf{G}) & \mathbf{M}^{-1} \mathbf{Y}_{1}(t) & \mathbf{M}^{-1} \mathbf{Y}_{2}(t) \\
\mathbf{0} & \mathbf{0} & \mathbf{A}_{1}(t) & \mathbf{0} \\
\mathbf{0} & \mathbf{0} & \mathbf{0} & \mathbf{A}_{\mathbf{1}}(t)
\end{array}\right]}_{\mathbf{A}(t)} \\
& \mathbf{y}=\underbrace{\left[\begin{array}{ll}
\mathbf{I}_{4 \times 4} & \mathbf{0}_{4 \times 12}
\end{array}\right]}_{\mathbf{B}} \mathbf{Z}
\end{aligned}
$$

where $\mathbf{z}=\left[\begin{array}{llll}\mathbf{q}^{T} & \dot{\mathbf{q}}^{T} & \mathbf{g}_{1}^{T} & \mathbf{g}_{2}^{T}\end{array}\right]^{T}$ is a augmented state, $\mathbf{y}=\mathbf{q}, \mathbf{B}$ is a constant matrix, $\mathbf{Y}_{\mathbf{1}}(t)$ and $\mathbf{Y}_{2}(t)$ are defined as

$$
\mathbf{Y}_{1}(t)=\left[\begin{array}{cccc}
0 & -\ddot{\theta} & 0 & -\dot{\theta} \\
\ddot{\theta} & 0 & \dot{\theta} & 0 \\
0 & 0 & 0 & 0 \\
0 & 0 & 0 & 0
\end{array}\right] ; \mathbf{Y}_{2}(t)=\left[\begin{array}{cccc}
0 & 0 & 0 & 0 \\
0 & 0 & 0 & 0 \\
0 & -\ddot{\theta} & 0 & -\dot{\theta} \\
\ddot{\theta} & 0 & \dot{\theta} & 0
\end{array}\right]
$$

Using this modified model, the state estimation problem can be used for different speed laws. As shown in the state space model, displacement can be measured, however, the implicitly included unbalances $U_{i}$ cannot be measured directly. An observer will used to estimate the states from incomplete states measurements.

\section{Time Varying Observer}

The rigid dynamic system presented in the state space model as

$$
\begin{gathered}
\dot{\mathbf{z}}(t)=\mathbf{A}(t) \mathbf{z}(t) \\
\mathbf{y}(t)=\mathbf{B z}(t)
\end{gathered}
$$

Introduce a state estimator in the following form

$$
\dot{\hat{\mathbf{z}}}(t)=\mathbf{F}(t) \hat{\mathbf{z}}(t)+\mathbf{G}(t) \mathbf{y}(t)
$$

where $\hat{\mathbf{z}}(t)$ is the estimated state vector, it has been proven by the Identity State Estimator Lemma, if $\mathbf{F}=\mathbf{A}(t)-\mathbf{G}(t) \mathbf{B}$ is a constant matrix with stable eigenvalues, then the system described by (11) is an asymptotic identity state estimator [5]. Then the relation of the true state $\mathbf{z}$ and its estimated state $\hat{\mathbf{z}}$ can be obtained

$$
\dot{\hat{\mathbf{z}}}-\dot{\mathbf{z}}=\mathbf{F}(\hat{\mathbf{z}}-\mathbf{z})
$$

The design of asymptotic state estimators procedures are described as follows:

- Obtain a Lyapunov transformation matrix $\mathbf{P}(t)$ by using the method stated in [5].

- Compute $\overline{\mathbf{A}}(t)=\mathbf{P}^{-1}(t)[\mathbf{A}(t) \mathbf{P}(t)-\dot{\mathbf{P}}(t)]$ and $\overline{\mathbf{B}}(t)=\mathbf{B P}(t)$, it has been shown in [5] that the matrix $\overline{\mathbf{A}}(t)$ and $\overline{\mathbf{B}}(t)$ are canonical forms. Now the original state space model (10) has been transformed into canonical structures

$$
\begin{aligned}
\dot{\overline{\mathbf{z}}}(t) & =\overline{\mathbf{A}}(t) \overline{\mathbf{z}}(t) \\
\mathbf{y}(t) & =\overline{\mathbf{B}}(t) \overline{\mathbf{z}}(t)
\end{aligned}
$$


- Choose distinct desired eigenvalues to design matrix $\overline{\mathbf{F}}$ as stated in [3], $\overline{\mathbf{F}}$ is a constant matrix with stable eigenvalues.

- Using the relation $\overline{\mathbf{F}}=\overline{\mathbf{A}}(t)-\overline{\mathbf{G}}(t) \overline{\mathbf{B}}(t)$ to obtain $\overline{\mathbf{G}}(t)$.

- Hence, an asymptotic identity state estimator of the equivalent system (13) is given by

$$
\overline{\mathbf{z}}(t)=\overline{\mathbf{F}} \overline{\mathbf{z}}(t)+\overline{\mathbf{G}}(t) \mathbf{y}(t)
$$

where $\overline{\mathbf{z}}(t)$ is the equivalent estimated state, $\mathbf{y}(t)$ is the output of original system (8).

- The original estimated state is obtained by $\hat{\mathbf{z}}=\mathbf{P}(t) \overline{\mathbf{z}}(t)$.

Following the above procedures, a desired time-varying observer for the rigid rotor can be design. The estimation error will go to zero as $t \rightarrow \infty$. So the estimation of implicitly included unbalances can be obtained.

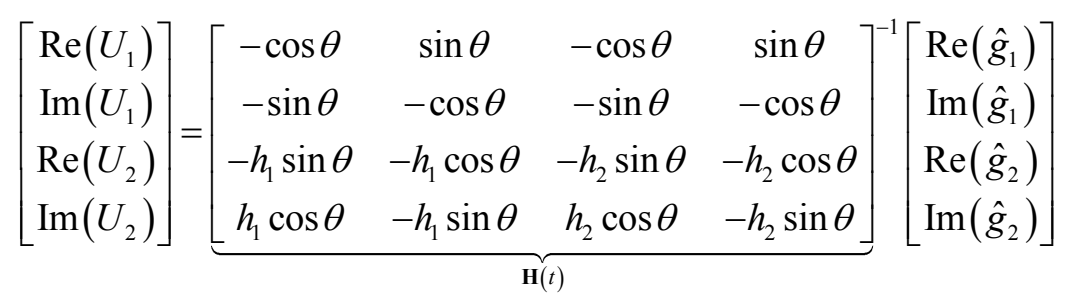

Since $\operatorname{det}(\mathbf{H})=\left(h_{1}-h_{2}\right)^{2}$ is always positive unless the two unbalance correction plans coincide, which can be avoid by proper choosing the location of the correction plans.

\section{Simulation}

A simulation is presented to illustrate the above procedures and methods. The rigid rotor shown as figure 1 has a length of $0.5 \mathrm{~m}$ and a diameter of $0.2 \mathrm{~m}$ and is made from steel with a density of $7810 \mathrm{~kg} / \mathrm{m}^{3}$, the locations in the rotation axis are $l_{1}=-0.4 \mathrm{~m}, l_{2}=0.3 \mathrm{~m}, h_{1}=-0.2 \mathrm{~m}$ and $h_{2}=0.2 \mathrm{~m}$. The unbalances are set to $(-0.03+j * 0.01) \mathrm{kgm}$ at plan I and $(0.05+j * 0.04) \mathrm{kgm}$ at plan II. The stiffness in both directions are $1 \mathrm{MN} / \mathrm{m}$ at bearing 1 and 1.3 $\mathrm{MN} / \mathrm{m}$ at bearing 2 . The damping values in in both directions are $100 \mathrm{Ns} / \mathrm{m}$ at bearing 1 and $130 \mathrm{Ns} / \mathrm{m}$ at bearing 2. The critical speeds of the rigid rotor are calculated as $21.79 \mathrm{~Hz}, 21.79 \mathrm{~Hz}, 44.93 \mathrm{~Hz}$ and $55.85 \mathrm{~Hz}$.

The unbalance estimation under linear acceleration is implement at first, the angular acceleration is set as a constant value of $100 \mathrm{rad} / \mathrm{s}^{2}$, as shown in figure 2, the true values of the unbalances in the two correction plan are shown by dashed lines, the solid lines are the estimated results which converges to the inherent unbalances quickly. 


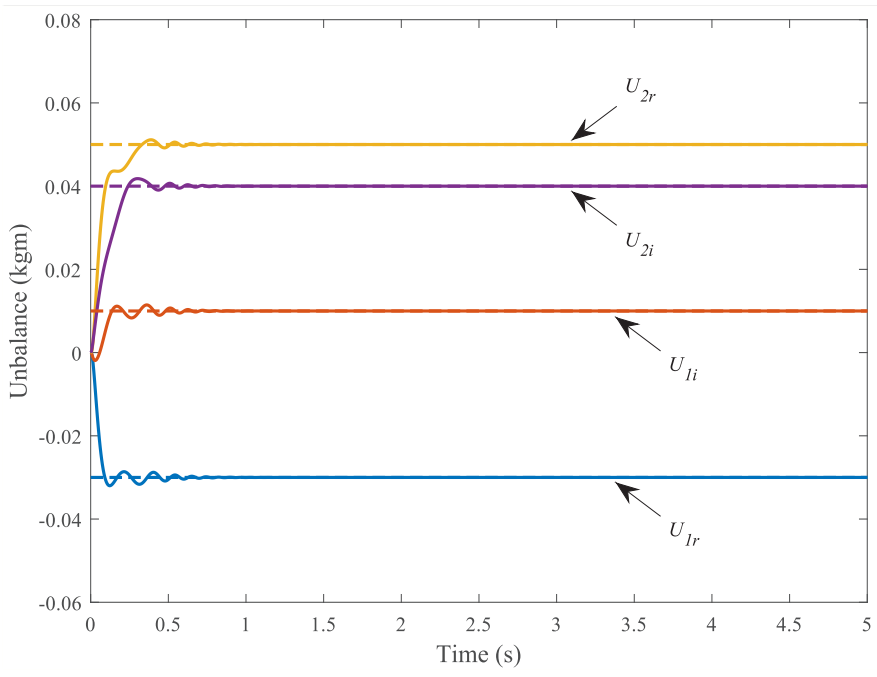

Figure 2. Estimated result under linear acceleration.

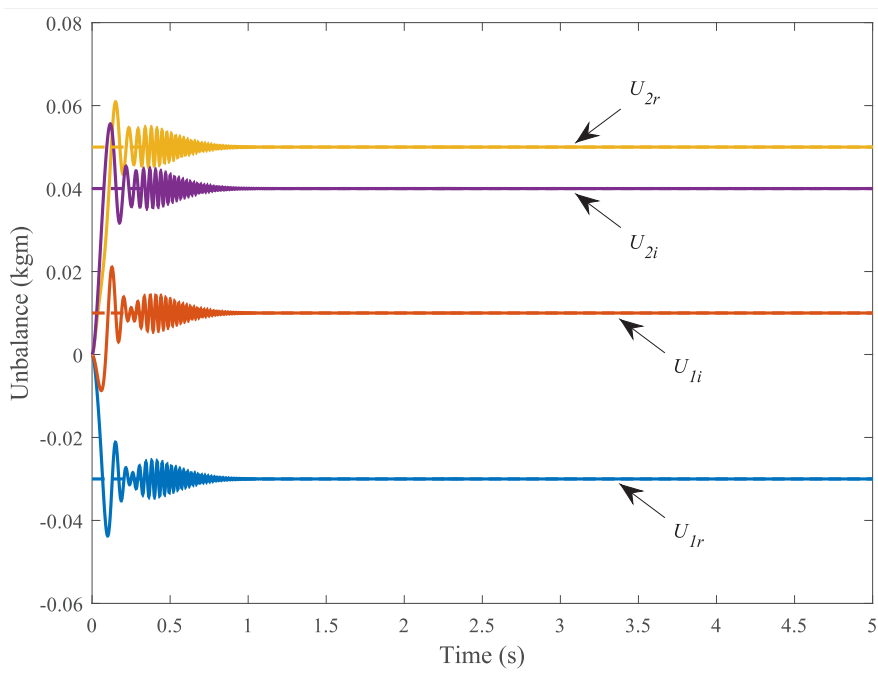

Figure 3. Estimated result under exponential acceleration.

Then another common speed law is used for test, the angular speed is exponential accelerated as $1000 *\left(1-e^{-0.5 t}\right) \mathrm{rad} / \mathrm{s}$, the great angular acceleration in the beginning may excite undesired transient response as shown in figure 3 , which results that the estimation oscillate around its true values, however, after passing this region the estimated results also converges to the inherent unbalances quickly. Those results prove that the extended time-varying observer works well for different acceleration law, and the performance is desire for active balancing.

\section{Conclusions}

This work aims to extend the method of applying time-varying observer for unbalance estimation in order to improving the adaption for different spin acceleration law. By assuming the dynamic parameters are known and the displacements and spin speed can be measured, the unbalances are viewing as the outputs of states of the dynamic rigid rotor system, then a new time-varying observer 
obtained without constant angular speed acceleration assumption. The simulation results show that the estimation converges to the inherent unbalances quickly under different law of acceleration.

\section{Funding}

This work is supported by the Hi-Tech Research and Development Program (863) of China (Grant NO. 2015AA043302).

\section{References}

1. Knospe C R and Tamer S M 1997 Experiments in robust control of rotor unbalance response using magnetic bearings Mechatronics 7 217-29

2. Zhou S, Dyer S W, Shin K-k, Shi J and Ni J 2004 Extended Influence Coefficient Method for Rotor Active Balancing During Acceleration Journal of Dynamic Systems, Measurement, and Control 126 219-23

3. Zhou S Y and Shi J J 2001 Imbalance estimation for speed-varying rigid rotors using timevarying observer J Dyn Syst-T Asme 123 637-44

4. Genta G 2005 Dynamics of rotating systems (New York: Springer)

5. Cuong N and Ting L 1985 Design of a state estimator for a class of time-varying multivariable systems IEEE Transactions on Automatic Control 30 179-82 